Submitted 18-Nov-2010; accepted 28-Oct-2011;

to Applied Mathematics and Computation (ISSN: 0096-3003).

In press, DOI: 10.1016/j.amc.2011.10.075 (http://dx.doi.org/10.1016/j.amc.2011.10.075)

\title{
MULTIOBJECTIVE FRACTIONAL VARIATIONAL CALCULUS IN TERMS OF A COMBINED CAPUTO DERIVATIVE*
}

\author{
AGNIESZKA B. MALINOWSKA ${ }^{\dagger}$ AND DELFIM F. M. TORRES ${ }^{\ddagger}$
}

\begin{abstract}
The study of fractional variational problems in terms of a combined fractional Caputo derivative is introduced. Necessary optimality conditions of Euler-Lagrange type for the basic, isoperimetric, and Lagrange variational problems are proved, as well as transversality and sufficient optimality conditions. This allows to obtain necessary and sufficient Pareto optimality conditions for multiobjective fractional variational problems.
\end{abstract}

Key words. Variational analysis, multiobjective optimization, fractional Euler-Lagrange equations, fractional derivatives in the sense of Caputo, Pareto minimizers.

AMS subject classifications. 49K05, 26A33.

1. Introduction. There is an increasing interest in the study of dynamic systems of fractional (where "fractional" actually means "non-integer") order. Extending derivatives and integrals from integer to non-integer order has a firm and longstanding theoretical foundation. Leibniz mentioned this concept in a letter to L'Hopital over three hundred years ago. Following L'Hopital's and Leibniz's first inquisition, fractional calculus was primarily a study reserved to the best minds in mathematics. Fourier, Euler, and Laplace are among the many that contributed to the development of fractional calculus. Along the history, many found, using their own notation and methodology, definitions that fit the concept of a non-integer order integral or derivative. The most famous of these definitions among mathematicians, that have been popularized in the literature of fractional calculus, are the ones of Riemann-Liouville and Grunwald-Letnikov. On the other hand, the most intriguing and useful applications of fractional derivatives and integrals in engineering and science have been found in the past one hundred years. In some cases, the mathematical notions evolved in order to better meet the requirements of physical reality. The best example of this is the Caputo fractional derivative, nowadays the most popular fractional operator among engineers and applied scientists, obtained by reformulating the "classical" definition of Riemann-Liouville fractional derivative in order to be possible to solve fractional initial value problems with standard initial conditions 34. Particularly in the last decade of the XX century, numerous applications and physical manifestations of fractional calculus have been found. Fractional differentiation is nowadays recognized as a good tool in various different fields: physics, signal processing, fluid mechanics, viscoelasticity, mathematical biology, electrochemistry, chemistry, economics, engineering, and control theory (see, e.g., $9,10,14,19,20,23,25,30,37,44,47$ ).

The fractional calculus of variations was born in 1996 with the work of Riewe [39, 40, and is nowadays a subject under strong current research (see [2, 3, 7, 8, 11, 12,

${ }^{*}$ This work was partially supported by the Portuguese Foundation for Science and Technology through the R\&D unit Center for Research and Development in Mathematics and Applications.

${ }^{\dagger}$ Faculty of Computer Science, Białystok University of Technology, 15-351 Białystok, Poland (a.malinowska@pb.edu.pl). Partially supported by BUT Grant S/WI/2/2011.

${ }^{\ddagger}$ Department of Mathematics, University of Aveiro, 3810-193 Aveiro, Portugal (delfim@ua.pt). Partially supported by FCT (Portugal) through the project UTAustin/MAT/0057/2008. 
15, 17, 35 and references therein). The fractional calculus of variations extends the classical variational calculus by considering fractional derivatives into the variational integrals to be extremized. This occurs naturally in many problems of physics and mechanics, in order to provide more accurate models of physical phenomena (see, e.g., 1, 5]). The aims of this paper are twofold. Firstly, we extend the notion of Caputo fractional derivative to the fractional derivative ${ }^{C} D_{\gamma}^{\alpha, \beta}$, which is a convex combination of the left Caputo fractional derivative of order $\alpha$ and the right Caputo fractional derivative of order $\beta$. This idea goes back at least as far as [22, where based on the Riemann-Liouville fractional derivatives, the symmetric fractional derivative was introduced. Klimek's approach [22] is obtained in our framework as a particular case, by choosing parameter $\gamma$ to be $1 / 2$. Although the symmetric fractional derivative of Riemann-Liouville introduced by Klimek is a useful tool in the description of some nonconservative models, this type of differentiation does not seems suitable for all kinds of variational problems. Indeed, the hypothesis that admissible trajectories $y$ have continuous symmetric fractional derivatives implies that $y(a)=y(b)=0$ (cf. [1]). Therefore, the advantage of the fractional Caputo-type derivative ${ }^{C} D_{\gamma}^{\alpha, \beta}$ here introduced lies in the fact that using this derivative we can describe a more general class of variational problems. It is also worth pointing out that the fractional derivative ${ }^{C} D_{\gamma}^{\alpha, \beta}$ allows to generalize the results presented in [4. Our second aim is to introduce the subject of multiobjective fractional variational problems. This seems to be a completely open area of research, never considered before in the literature. Knowing the importance and relevance of multiobjective problems of the calculus of variations in physics, engineering, and economics (see [13, 26, 27, 33, 43, 46, and the references given there), and the usefulness of fractional variational problems, we trust that the results now obtained will open interesting possibilities for future research. Main results of the the paper provide methods for identifying Pareto optimal solutions. Necessary and sufficient Pareto optimality conditions are obtained by converting a multiobjective fractional variational problem into a single or a family of single fractional variational problems with an auxiliary scalar functional, possibly depending on some parameters.

The paper is organized as follows. Section 2 presents some preliminaries on fractional calculus, essentially to fix notations. In Section 3 we introduce the fractional derivative ${ }^{C} D_{\gamma}^{\alpha, \beta}$ and provide the necessary concepts and results needed in the sequel. Our main results are stated and proved in Section 4 and Section 5 The fractional variational problems under our consideration are formulated in terms of the fractional derivative ${ }^{C} D_{\gamma}^{\alpha, \beta}$. We discuss the fundamental concepts of a variational calculus such as the the Euler-Lagrange equations for the elementary (Subsection4.1), isoperimetric (Subsection 4.3), and Lagrange (Subsection 4.4) problems, as well as sufficient optimality (Subsection 4.5) and transversality (Subsection 4.2) conditions. Section 5]deals with the multiobjective fractional variational calculus. We present Pareto optimality conditions (Subsection [5.1) and examples illustrating our results (Subsection [5.2).

2. Fractional calculus. In this section we review the necessary definitions and facts from fractional calculus. For more on the subject we refer the reader to the books [21,36, 38, 42. Let $f \in L_{1}([a, b])$ and $0<\alpha<1$. We begin by defining the left and the right Riemann-Liouville Fractional Integrals (RLFI) of order $\alpha$ of a function $f$. The left RLFI is given by

$$
{ }_{a} I_{x}^{\alpha} f(x):=\frac{1}{\Gamma(\alpha)} \int_{a}^{x}(x-t)^{\alpha-1} f(t) d t, \quad x \in[a, b],
$$


and the right RLFI by

$$
{ }_{x} I_{b}^{\alpha} f(x):=\frac{1}{\Gamma(\alpha)} \int_{x}^{b}(t-x)^{\alpha-1} f(t) d t, \quad x \in[a, b],
$$

where $\Gamma(\cdot)$ represents the Gamma function, i.e.,

$$
\Gamma(z):=\int_{0}^{\infty} t^{z-1} \mathrm{e}^{-t} d t
$$

Moreover, ${ }_{a} I_{x}^{0} f={ }_{x} I_{b}^{0} f=f$ if $f$ is a continuous function. The left and the right Riemann-Liouville derivatives are defined with the help of the respective fractional integrals. The left Riemann-Liouville Fractional Derivative (RLFD) is given by

$$
{ }_{a} D_{x}^{\alpha} f(x):=\frac{1}{\Gamma(1-\alpha)} \frac{d}{d x} \int_{a}^{x}(x-t)^{-\alpha} f(t) d t=\frac{d}{d x}{ }_{a} I_{x}^{1-\alpha} f(x), \quad x \in[a, b],
$$

and the right RLFD by

$$
{ }_{x} D_{b}^{\alpha} f(x):=\frac{-1}{\Gamma(1-\alpha)} \frac{d}{d x} \int_{x}^{b}(t-x)^{-\alpha} f(t) d t=\left(-\frac{d}{d x}\right){ }_{x} I_{b}^{1-\alpha} f(x), \quad x \in[a, b] .
$$

Let $f \in A C([a, b])$, where $A C([a, b])$ represents the space of absolutely continuous functions on $[a, b]$. Then the Caputo fractional derivatives are defined as follows: the left Caputo Fractional Derivative (CFD) by

$$
{ }_{a}^{C} D_{x}^{\alpha} f(x):=\frac{1}{\Gamma(1-\alpha)} \int_{a}^{x}(x-t)^{-\alpha} \frac{d}{d t} f(t) d t={ }_{a} I_{x}^{1-\alpha} \frac{d}{d x} f(x), \quad x \in[a, b],
$$

and the right $\mathrm{CFD}$ by

$$
{ }_{x}^{C} D_{b}^{\alpha} f(x):=\frac{-1}{\Gamma(1-\alpha)} \int_{x}^{b}(t-x)^{-\alpha} \frac{d}{d t} f(t) d t={ }_{x} I_{b}^{1-\alpha}\left(-\frac{d}{d x}\right) f(x), \quad x \in[a, b],
$$

where $\alpha$ is the order of the derivative. The operators (2.1)-(2.6) are obviously linear. We now present the rule of fractional integration by parts for RLFI (see, e.g., [24]). Let $0<\alpha<1, p \geq 1, q \geq 1$, and $1 / p+1 / q \leq 1+\alpha$. If $g \in L_{p}([a, b])$ and $f \in L_{q}([a, b])$, then

$$
\int_{a}^{b} g(x)_{a} I_{x}^{\alpha} f(x) d x=\int_{a}^{b} f(x)_{x} I_{b}^{\alpha} g(x) d x .
$$

In the discussion to follow, we will also need the following formulae for fractional integrations by parts:

$$
\begin{aligned}
& \int_{a}^{b} g(x){ }_{a}^{C} D_{x}^{\alpha} f(x) d x=\left.f(x)_{x} I_{b}^{1-\alpha} g(x)\right|_{x=a} ^{x=b}+\int_{a}^{b} f(x)_{x} D_{b}^{\alpha} g(x) d x, \\
& \int_{a}^{b} g(x)_{x}^{C} D_{b}^{\alpha} f(x) d x=-\left.f(x)_{a} I_{x}^{1-\alpha} g(x)\right|_{x=a} ^{x=b}+\int_{a}^{b} f(x)_{a} D_{x}^{\alpha} g(x) d x .
\end{aligned}
$$

They can be derived using equations (2.3)-(2.6), the identity (2.7), and performing integration by parts. 
3. The fractional operator ${ }^{\mathbf{C}} \mathbf{D}_{\gamma}^{\alpha, \beta}$. Let $\alpha, \beta \in(0,1)$ and $\gamma \in[0,1]$. We define the fractional derivative operator ${ }^{C} D_{\gamma}^{\alpha, \beta}$ by

$$
{ }^{C} D_{\gamma}^{\alpha, \beta}:=\gamma{ }_{a}^{C} D_{x}^{\alpha}+(1-\gamma){ }_{x}^{C} D_{b}^{\beta},
$$

which acts on $f \in A C([a, b])$ in the expected way:

$$
{ }^{C} D_{\gamma}^{\alpha, \beta} f(x)=\gamma_{a}^{C} D_{x}^{\alpha} f(x)+(1-\gamma){ }_{x}^{C} D_{b}^{\beta} f(x) .
$$

Note that ${ }^{C} D_{0}^{\alpha, \beta} f(x)={ }_{x}^{C} D_{b}^{\beta} f(x)$ and ${ }^{C} D_{1}^{\alpha, \beta} f(x)={ }_{a}^{C} D_{x}^{\alpha} f(x)$. The operator (3.1) is obviously linear. Using equations (2.8) it is easy to derive the following rule of fractional integration by parts for ${ }^{C} D_{\gamma}^{\alpha, \beta}$ :

$$
\begin{aligned}
\int_{a}^{b} g(x){ }^{C} D_{\gamma}^{\alpha, \beta} f(x) d x & =\gamma\left[f(x)_{x} I_{b}^{1-\alpha} g(x)\right]_{x=a}^{x=b} \\
+ & (1-\gamma)\left[-f(x)_{a} I_{x}^{1-\beta} g(x)\right]_{x=a}^{x=b}+\int_{a}^{b} f(x) D_{1-\gamma}^{\beta, \alpha} g(x) d x,
\end{aligned}
$$

where $D_{1-\gamma}^{\beta, \alpha}:=(1-\gamma)_{a} D_{x}^{\beta}+\gamma_{x} D_{b}^{\alpha}$. Let $N \in \mathbb{N}$ and $\mathbf{f}=\left[f_{1}, \ldots, f_{N}\right]:[a, b] \rightarrow \mathbb{R}^{N}$ with $f_{i} \in A C([a, b]), i=1, \ldots, N ; \alpha, \beta, \gamma \in \mathbb{R}^{N}$ with $\alpha_{i}, \beta_{i} \in(0,1)$ and $\gamma_{i} \in[0,1]$, $i=1, \ldots, N$. Then,

$$
{ }^{C} D_{\gamma}^{\alpha, \beta} \mathbf{f}(x):=\left[{ }^{C} D_{\gamma_{1}}^{\alpha_{1}, \beta_{1}} f_{1}(x), \ldots,{ }^{C} D_{\gamma_{N}}^{\alpha_{N}, \beta_{N}} f_{N}(x)\right] .
$$

Let $\mathbf{D}$ denote the set of all functions $\mathbf{y}:[a, b] \rightarrow \mathbb{R}^{N}$ such that ${ }^{C} D_{\gamma}^{\alpha, \beta} \mathbf{y}$ exists and is continuous on the interval $[a, b]$. We endow $\mathbf{D}$ with the following norm:

$$
\|\mathbf{y}\|_{1, \infty}:=\max _{a \leq x \leq b}\|\mathbf{y}(x)\|+\max _{a \leq x \leq b}\left\|^{C} D_{\gamma}^{\alpha, \beta} \mathbf{y}(x)\right\|,
$$

where $\|\cdot\|$ is a norm in $\mathbb{R}^{N}$. Along the work we denote by $\partial_{i} K, i=1, \ldots, M(M \in \mathbb{N})$, the partial derivative of function $K: \mathbb{R}^{M} \rightarrow \mathbb{R}$ with respect to its $i$ th argument. Let $\lambda \in \mathbb{R}^{r}$. For simplicity of notation we introduce the operators $[\mathbf{y}]_{\gamma}^{\alpha, \beta}$ and $\lambda_{\lambda}\{\mathbf{y}\}_{\gamma}^{\alpha, \beta}$ by

$$
\begin{aligned}
{[\mathbf{y}]_{\gamma}^{\alpha, \beta}(x) } & :=\left(x, \mathbf{y}(x),{ }^{C} D_{\gamma}^{\alpha, \beta} \mathbf{y}(x)\right), \\
\lambda\{\mathbf{y}\}_{\gamma}^{\alpha, \beta}(x) & :=\left(x, \mathbf{y}(x),{ }^{C} D_{\gamma}^{\alpha, \beta} \mathbf{y}(x), \lambda_{1}, \ldots, \lambda_{r}\right) .
\end{aligned}
$$

4. Calculus of variations via ${ }^{\mathrm{C}} \mathbf{D}_{\gamma}^{\alpha, \beta}$. We are concerned with the problem of finding the minimum of a functional $\mathcal{J}: \mathcal{D} \rightarrow \mathbb{R}$, where $\mathcal{D}$ is a subset of $\mathbf{D}$. The formulation of a problem of the calculus of variations requires two steps: the specification of a performance criterion, and the statement of physical constraints that should be satisfied. The performance criterion $\mathcal{J}$, also called cost functional (or objective), must be specified for evaluating quantitatively the performance of the system under study. We consider the following cost:

$$
\mathcal{J}(\mathbf{y})=\int_{a}^{b} L[\mathbf{y}]_{\gamma}^{\alpha, \beta}(x) d x
$$


where $x \in[a, b]$ is the independent variable; $\mathbf{y}(x) \in \mathbb{R}^{N}$ is a real vector variable, the functions $\mathbf{y}$ are generally called trajectories or curves; ${ }^{C} D_{\gamma}^{\alpha, \beta} \mathbf{y}(x) \in \mathbb{R}^{N}$ stands for the fractional derivative of $\mathbf{y}(x)$; and $L \in C^{1}\left([a, b] \times \mathbb{R}^{2 N} ; \mathbb{R}\right)$ is the Lagrangian.

Enforcing constraints in the optimization problem reduces the set of candidate functions and leads to the following definition.

Definition 4.1. A trajectory $\mathbf{y} \in \mathbf{D}$ is said to be an admissible trajectory, provided it satisfies all the constraints of the problem along the interval $[a, b]$. The set of admissible trajectories is defined as $\mathcal{D}:=\{\mathbf{y} \in \mathbf{D}: \mathbf{y}$ is admissible $\}$.

We now define what is meant by a minimum of $\mathcal{J}$ on $\mathcal{D}$.

Definition 4.2. A trajectory $\overline{\mathbf{y}} \in \mathcal{D}$ is said to be a local minimizer for $\mathcal{J}$ on $\mathcal{D}$, if there exists $\delta>0$ such that $\mathcal{J}(\overline{\mathbf{y}}) \leq \mathcal{J}(\mathbf{y})$ for all $\mathbf{y} \in \mathcal{D}$ with $\|\mathbf{y}-\overline{\mathbf{y}}\|_{1, \infty}<\delta$.

The concept of variation of a functional is central to the solution of problems of the calculus of variations.

Definition 4.3. The first variation of $\mathcal{J}$ at $\mathbf{y} \in \mathbf{D}$ in the direction $\mathbf{h} \in \mathbf{D}$ is defined as

$$
\delta \mathcal{J}(\mathbf{y} ; \mathbf{h}):=\lim _{\varepsilon \rightarrow 0} \frac{\mathcal{J}(\mathbf{y}+\varepsilon \mathbf{h})-\mathcal{J}(\mathbf{y})}{\varepsilon}=\left.\frac{\partial}{\partial \varepsilon} \mathcal{J}(\mathbf{y}+\varepsilon \mathbf{h})\right|_{\varepsilon=0},
$$

provided the limit exists.

Definition 4.4. A direction $\mathbf{h} \in \mathbf{D}, \mathbf{h} \neq 0$, is said to be an admissible variation for $\mathcal{J}$ at $\mathbf{y} \in \mathcal{D}$ if

(i) $\delta \mathcal{J}(\mathbf{y} ; \mathbf{h})$ exists; and

(ii) $\mathbf{y}+\varepsilon \mathbf{h} \in \mathcal{D}$ for all sufficiently small $\varepsilon$.

The following well known result offers a necessary optimality condition for the problems of the calculus of variations, based on the concept of variations.

Theorem 4.5 (see, e.g., Proposition 5.5 of [45]). Let $\mathcal{J}$ be a functional defined on $\mathcal{D}$. Suppose that $\mathbf{y}$ is a local minimizer for $\mathcal{J}$ on $\mathcal{D}$. Then, $\delta \mathcal{J}(\mathbf{y} ; \mathbf{h})=0$ for each admissible variation $\mathbf{h}$ at $\mathbf{y}$.

4.1. Elementary problem of the ${ }^{\mathrm{C}} \mathbf{D}_{\gamma}^{\alpha, \beta}$ fractional calculus of variations. Let us begin with the following fundamental problem:

$$
\mathcal{J}(\mathbf{y})=\int_{a}^{b} L[\mathbf{y}]_{\gamma}^{\alpha, \beta}(x) d x \longrightarrow \min
$$

over all $\mathbf{y} \in \mathbf{D}$ satisfying the boundary conditions

$$
\mathbf{y}(a)=\mathbf{y}^{a}, \quad \mathbf{y}(b)=\mathbf{y}^{b},
$$

where $\mathbf{y}^{a}, \mathbf{y}^{b} \in \mathbb{R}^{N}$ are given. The next theorem gives the fractional Euler-Lagrange equation for the problem (4.1)-(4.2).

THEOREM 4.6. Let $\mathbf{y}=\left(y_{1}, \ldots, y_{N}\right)$ be a local minimizer to problem (4.1)-(4.2). Then, $\mathbf{y}$ satisfies the following system of $N$ fractional Euler-Lagrange equations:

$$
\partial_{i} L[\mathbf{y}]_{\gamma}^{\alpha, \beta}(x)+D_{1-\gamma_{i-1}}^{\beta_{i-1}, \alpha_{i-1}} \partial_{N+i} L[\mathbf{y}]_{\gamma}^{\alpha, \beta}(x)=0, \quad i=2, \ldots N+1,
$$

for all $x \in[a, b]$.

Proof. Suppose that $\mathbf{y}$ is a local minimizer for $\mathcal{J}$. Let $\mathbf{h}$ be an arbitrary admissible variation for problem (4.1)-(4.2), i.e., $h_{i}(a)=h_{i}(b)=0, i=1, \ldots, N$. Based on the 
differentiability properties of $L$ and Theorem 4.5, a necessary condition for $\mathbf{y}$ to be a local minimizer is given by

$$
\left.\frac{\partial}{\partial \varepsilon} \mathcal{J}(\mathbf{y}+\varepsilon \mathbf{h})\right|_{\varepsilon=0}=0
$$

that is,

$$
\int_{a}^{b}\left[\sum_{i=2}^{N+1} \partial_{i} L[\mathbf{y}]_{\gamma}^{\alpha, \beta}(x) h_{i-1}(x)+\sum_{i=2}^{N+1} \partial_{N+i} L[\mathbf{y}]_{\gamma}^{\alpha, \beta}(x)^{C} D_{\gamma_{i-1}}^{\alpha_{i-1}, \beta_{i-1}} h_{i-1}(x)\right] d x=0
$$

Using formulae (3.2) of integration by parts in the second term of the integrand function, we get

$$
\begin{aligned}
\int_{a}^{b}\left[\sum_{i=2}^{N+1} \partial_{i} L[\mathbf{y}]_{\gamma}^{\alpha, \beta}(x)+D_{1-\gamma_{i-1}}^{\beta_{i-1}, \alpha_{i-1}} \partial_{N+i} L[\mathbf{y}]_{\gamma}^{\alpha, \beta}(x)\right] h_{i-1}(x) d x \\
+\left.\gamma\left[\sum_{i=2}^{N+1} h_{i-1}(x)\left({ }_{x} I_{b}^{1-\alpha_{i-1}} \partial_{N+i} L[\mathbf{y}]_{\gamma}^{\alpha, \beta}(x)\right)\right]\right|_{x=a} ^{x=b} \\
-\left.(1-\gamma)\left[\sum_{i=2}^{N+1} h_{i-1}(x)\left({ }_{a} I_{x}^{1-\beta_{i-1}} \partial_{N+i} L[\mathbf{y}]_{\gamma}^{\alpha, \beta}(x)\right)\right]\right|_{x=a} ^{x=b}=0 .
\end{aligned}
$$

Since $h_{i}(a)=h_{i}(b)=0, i=1, \ldots, N$, by the fundamental lemma of the calculus of variations we deduce that

$$
\partial_{i} L[\mathbf{y}]_{\gamma}^{\alpha, \beta}(x)+D_{1-\gamma_{i-1}}^{\beta_{i-1}, \alpha_{i-1}} \partial_{N+i} L[\mathbf{y}]_{\gamma}^{\alpha, \beta}(x)=0, \quad i=2, \ldots, N+1,
$$

for all $x \in[a, b]$.

Observe that if $\alpha$ and $\beta$ go to 1 , then ${ }_{a}^{C} D_{x}^{\alpha}$ can be replaced with $\frac{d}{d x}$ and ${ }_{x}^{C} D_{b}^{\beta}$ with $-\frac{d}{d x}$ (see [38]). Thus, if $\gamma=1$ or $\gamma=0$, then for $\alpha, \beta \rightarrow 1$ we obtain a corresponding result in the classical context of the calculus of variations (see, e.g., [45, Proposition 6.1]).

4.2. Fractional transversality conditions. Let $l \in\{1, \ldots, N\}$. Assume that $\mathbf{y}(a)=\mathbf{y}^{a}, y_{i}(b)=y_{i}^{b}, i=1, \ldots, N, i \neq l$, but $y_{l}(b)$ is free. Then, $h_{l}(b)$ is free and by equations (4.3) and (4.5) we obtain

$$
\left.\left[\gamma_{x} I_{b}^{1-\alpha_{l}} \partial_{N+l+1} L[\mathbf{y}]_{\gamma}^{\alpha, \beta}(x)-(1-\gamma)_{a} I_{x}^{1-\beta_{l}} \partial_{N+1+l} L[\mathbf{y}]_{\gamma}^{\alpha, \beta}(x)\right]\right|_{x=b}=0
$$

where $\alpha, \beta, \gamma \in \mathbb{R}$. Let us consider now the case when $\mathbf{y}(a)=\mathbf{y}^{a}, y_{i}(b)=y_{i}^{b}$, $i=1, \ldots, N, i \neq l$, and $y_{l}(b)$ is free but restricted by a terminal condition $y_{l}(b) \leq y_{l}^{b}$. Then, in the optimal solution $\mathbf{y}$, we have two possible types of outcome: $y_{l}(b)<y_{l}^{b}$ or $y_{l}(b)=y_{l}^{b}$. If $y_{l}(b)<y_{l}^{b}$, then there are admissible neighboring paths with terminal value both above and below $y_{l}(b)$, so that $h_{l}(b)$ can take either sign. Therefore, the transversality conditions is

$$
\left.\left[\gamma_{x} I_{b}^{1-\alpha_{l}} \partial_{N+l+1} L[\mathbf{y}]_{\gamma}^{\alpha, \beta}(x)-(1-\gamma)_{a} I_{x}^{1-\beta_{l}} \partial_{N+1+l} L[\mathbf{y}]_{\gamma}^{\alpha, \beta}(x)\right]\right|_{x=b}=0
$$

for $y_{l}(b)<y_{l}^{b}$. The other outcome $y_{l}(b)=y_{l}^{b}$ only admits the neighboring paths with terminal value $\tilde{y}_{l}(b) \leq y_{l}(b)$. Assuming, without loss of generality, that $h_{l}(b) \geq 0$, this 
means that $\varepsilon \leq 0$. Hence, the transversality condition, which has it root in the first order condition (4.4), must be changed to an inequality. For a minimization problem, the $\leq$ type of inequality is called for, and we obtain

$$
\left.\left[\gamma_{x} I_{b}^{1-\alpha_{l}} \partial_{N+l+1} L[\mathbf{y}]_{\gamma}^{\alpha, \beta}(x)-(1-\gamma)_{a} I_{x}^{1-\beta_{l}} \partial_{N+1+l} L[\mathbf{y}]_{\gamma}^{\alpha, \beta}(x)\right]\right|_{x=b} \leq 0
$$

for $y_{l}(b)=y_{l}^{b}$. Combining (4.6) and (4.7), we may write the following transversality condition for a minimization problem:

$$
\begin{gathered}
{\left.\left[\gamma_{x} I_{b}^{1-\alpha_{l}} \partial_{N+l+1} L[\mathbf{y}]_{\gamma}^{\alpha, \beta}(x)-(1-\gamma)_{a} I_{x}^{1-\beta_{l}} \partial_{N+1+l} L[\mathbf{y}]_{\gamma}^{\alpha, \beta}(x)\right]\right|_{x=b} \leq 0, \quad y_{l}(b) \leq y_{l}^{b},} \\
\left.\left(y_{l}(b)-y_{l}^{b}\right)\left[\gamma_{x} I_{b}^{1-\alpha_{l}} \partial_{N+l+1} L[\mathbf{y}]_{\gamma}^{\alpha, \beta}(x)-(1-\gamma)_{a} I_{x}^{1-\beta_{l}} \partial_{N+1+l} L[\mathbf{y}]_{\gamma}^{\alpha, \beta}(x)\right]\right|_{x=b}=0 .
\end{gathered}
$$

4.3. The ${ }^{C} \mathbf{D}_{\gamma}^{\alpha, \beta}$ fractional isoperimetric problem. Let us consider now the isoperimetric problem that consists of minimizing (4.1) over all $\mathbf{y} \in \mathbf{D}$ satisfying $r$ isoperimetric constraints

$$
\mathcal{G}^{j}(\mathbf{y})=\int_{a}^{b} G^{j}[\mathbf{y}]_{\gamma}^{\alpha, \beta}(x) d x=l_{j}, \quad j=1, \ldots, r,
$$

where $G^{j} \in C^{1}\left([a, b] \times \mathbb{R}^{2 N} ; \mathbb{R}\right), j=1, \ldots, r$, and boundary conditions (4.2).

Necessary optimality conditions for isoperimetric problems can be obtained by the following general theorem.

Theorem 4.7 (see, e.g., Theorem 2 of [18] on p. 91). Let $\mathcal{J}, \mathcal{G}^{1}, \ldots, \mathcal{G}^{r}$ be functionals defined in a neighborhood of $\mathbf{y}$ and having continuous first variations in this neighborhood. Suppose that $\mathbf{y}$ is a local minimizer of (4.1) subject to the boundary conditions (4.2) and the isoperimetric constrains (4.8). Assume that there are functions $\mathbf{h}^{1}, \ldots, \mathbf{h}^{r} \in \mathbf{D}$ such that the matrix $A=\left(a_{k l}\right), a_{k l}:=\delta \mathcal{G}^{k}\left(\mathbf{y} ; \mathbf{h}^{l}\right)$, has maximal rank $r$. Then there exist constants $\lambda_{1}, \ldots, \lambda_{r} \in \mathbb{R}$ such that the functional

$$
\mathcal{F}:=\mathcal{J}-\sum_{j=1}^{r} \lambda_{j} \mathcal{G}^{j}
$$

satisfies

$$
\delta \mathcal{F}(\mathbf{y} ; \mathbf{h})=0
$$

for all $\mathbf{h} \in \mathbf{D}$

Suppose now that assumptions of Theorem 4.7 hold. Then, equation (4.9) is fulfilled for every $\mathbf{h} \in \mathbf{D}$. Let us consider function $\mathbf{h}$ such that $\mathbf{h}(a)=\mathbf{h}(b)=0$. Then, we have

$$
\begin{aligned}
& 0=\delta \mathcal{F}(\mathbf{y} ; \mathbf{h})=\left.\frac{\partial}{\partial \varepsilon} \mathcal{F}(\mathbf{y}+\varepsilon \mathbf{h})\right|_{\varepsilon=0} \\
= & \int_{a}^{b}\left[\sum_{i=2}^{N+1} \partial_{i} F_{\lambda}\{\mathbf{y}\}_{\gamma}^{\alpha, \beta}(x) h_{i-1}(x)+\sum_{i=2}^{N+1} \partial_{N+i} F_{\lambda}\{\mathbf{y}\}_{\gamma}^{\alpha, \beta}(x)^{C} D_{\gamma_{i-1}}^{\alpha_{i-1}, \beta_{i-1}} h_{i-1}(x)\right] d x,
\end{aligned}
$$

where the function $F:[a, b] \times \mathbb{R}^{2 N} \times \mathbb{R}^{r} \rightarrow \mathbb{R}$ is defined by

$$
F_{\lambda}\{\mathbf{y}\}_{\gamma}^{\alpha, \beta}(x):=L[\mathbf{y}]_{\gamma}^{\alpha, \beta}(x)-\sum_{j=1}^{r} \lambda_{j} G^{j}[\mathbf{y}]_{\gamma}^{\alpha, \beta}(x) .
$$


On account of the above, and similarly in spirit to the proof of Theorem4.6, we obtain

$$
\partial_{i \lambda} F\{\mathbf{y}\}_{\gamma}^{\alpha, \beta}(x)+D_{1-\gamma_{i-1}}^{\beta_{i-1}, \alpha_{i-1}} \partial_{N+i \lambda} F\{\mathbf{y}\}_{\gamma}^{\alpha, \beta}(x)=0, \quad i=2, \ldots N+1 .
$$

Therefore, we have the following necessary optimality condition for the fractional isoperimetric problems:

THEOREM 4.8. Let assumptions of Theorem 4.7 hold. If $\mathbf{y}$ is a local minimizer to the isoperimetric problem given by (4.1), (4.2) and (4.8), then $\mathbf{y}$ satisfies the system of $N$ fractional Euler-Lagrange equations (4.10) for all $x \in[a, b]$.

Suppose now that constraints (4.8) are characterized by inequalities

$$
\mathcal{G}^{j}(\mathbf{y})=\int_{a}^{b} G^{j}[\mathbf{y}]_{\gamma}^{\alpha, \beta}(x) d x \leq l_{j}, \quad j=1, \ldots, r .
$$

In this case we can set

$$
\int_{a}^{b}\left(G^{j}[\mathbf{y}]_{\gamma}^{\alpha, \beta}(x)-\frac{l_{j}}{b-a}\right) d x+\int_{a}^{b}\left(\phi_{j}(x)\right)^{2} d x=0,
$$

$j=1, \ldots, r$, where $\phi_{j}$ have the some continuity properties as $y_{i}$. Therefore, we obtain the following problem:

$$
\hat{\mathcal{J}}(y)=\int_{a}^{b} \hat{L}\left(x, \mathbf{y}(x),{ }^{C} D_{\gamma}^{\alpha, \beta} \mathbf{y}(x), \phi_{1}(x), \ldots, \phi_{r}(x)\right) d x \longrightarrow \min
$$

subject to $r$ isoperimetric constraints

$$
\int_{a}^{b}\left[G^{j}[\mathbf{y}]_{\gamma}^{\alpha, \beta}(x)-\frac{l_{j}}{b-a}+\left(\phi_{j}(x)\right)^{2}\right] d x=0, \quad j=1, \ldots, r,
$$

and boundary conditions (4.2). Assuming that assumptions of Theorem 4.8 are satisfied, we conclude that there exist constants $\lambda_{j} \in \mathbb{R}, j=1, \ldots, r$, for which the system of equations

$$
\begin{gathered}
D_{1-\gamma_{i-1}}^{\beta_{i-1}, \alpha_{i-1}} \partial_{N+i} \hat{F}\left(x, \mathbf{y}(x),{ }^{C} D_{\gamma}^{\alpha, \beta} \mathbf{y}(x), \lambda_{1}, \ldots, \lambda_{r}, \phi_{1}(x), \ldots, \phi_{r}(x)\right) \\
+\partial_{i} \hat{F}\left(x, \mathbf{y}(x),{ }^{C} D_{\gamma}^{\alpha, \beta} \mathbf{y}(x), \lambda_{1}, \ldots, \lambda_{r}, \phi_{1}(x), \ldots, \phi_{r}(x)\right)=0, \\
i=2, \ldots, N+1, \hat{F}=\hat{L}+\sum_{j=1}^{r} \lambda_{j}\left(G^{j}-\frac{l_{j}}{b-a}+\phi_{j}^{2}\right) \text { and } \\
\lambda_{j} \phi_{j}(x)=0, \quad j=1, \ldots, r,
\end{gathered}
$$

hold for all $x \in[a, b]$. Note that it is enough to assume that the regularity condition holds for the constraints which are active at the local minimizer y (constraint $\mathcal{G}^{k}$ is active at $\mathbf{y}$ if $\mathcal{G}^{k}(\mathbf{y})=l_{k}$ ). Indeed, suppose that $l<r$ constrains, say $\mathcal{G}^{1}, \ldots, \mathcal{G}^{l}$ for simplicity, are active at the local minimizer $\mathbf{y}$, and there are functions $\mathbf{h}^{1}, \ldots, \mathbf{h}^{l} \in \mathbf{D}$ such that the matrix

$$
B=\left(b_{k j}\right), \quad b_{k j}:=\delta \mathcal{G}^{k}\left(\mathbf{y} ; \mathbf{h}^{j}\right), \quad k, j=1, \ldots, l<r
$$

has maximal rank $l$. Since the inequality constraints $\mathcal{G}^{l+1}, \ldots, \mathcal{G}^{r}$ are inactive, the condition (4.12) is trivially satisfied by taking $\lambda_{l+1}=\cdots=\lambda_{r}=0$. On the other hand, since the inequality constraints $\mathcal{G}^{1}, \ldots, \mathcal{G}^{l}$ are active and satisfy a regularity condition at $\mathbf{y}$, the conclusion that there exist constants $\lambda_{j} \in \mathbb{R}, j=1, \ldots, r$, such that (4.11) holds follow from Theorem 4.8. Moreover, (4.12) is trivially satisfied for $j=1, \ldots, l$. 
4.4. The ${ }^{\mathbf{C}} \mathbf{D}_{\gamma}^{\alpha, \beta}$ fractional Lagrange problem. Let us consider the following Lagrange problem, which consists of minimizing (4.1) over all $\mathbf{y} \in \mathbf{D}$ satisfying $r$ independent constraints $(r<N)$

$$
G^{j}[\mathbf{y}]_{\gamma}^{\alpha, \beta}(x)=0, \quad j=1, \ldots, r,
$$

and boundary conditions (4.2). In mechanics, constraints of type (4.13) are called nonholonomic. By the independence of the $r$ constraints $G^{j} \in C^{1}\left([a, b] \times \mathbb{R}^{2 N} ; \mathbb{R}\right)$ it is meant that it should exist a nonvanishing Jacobian determinant of order $r$, such as $\left|\frac{\partial\left(G^{1}, \ldots, G^{r}\right)}{\partial\left(p_{N+2}, \ldots, p_{N+2+r}\right)}\right| \neq 0$. Of course, any $r$ of $p_{j}, j=N+2, \ldots, 2 N+1$, can be used, not necessarily the first $r$.

THeOREM 4.9. A function $\mathbf{y}$ which is a solution to problem (4.1), (4.2) subject to $r$ independent constraints $(r<N)$ (4.13) satisfies, for suitably chosen functions $\lambda_{j}$, $j=1, \ldots, r$, the system of $N$ fractional Euler-Lagrange equations

$$
\partial_{i} F[\mathbf{y}, \lambda]_{\gamma}^{\alpha, \beta}(x)+D_{1-\gamma_{i-1}}^{\beta_{i-1}, \alpha_{i-1}} \partial_{N+i} F[\mathbf{y}, \lambda]_{\gamma}^{\alpha, \beta}(x)=0, \quad x \in[a, b], \quad i=2, \ldots, N+1,
$$

where $F[\mathbf{y}, \lambda]_{\gamma}^{\alpha, \beta}(x)=L[\mathbf{y}]_{\gamma}^{\alpha, \beta}(x)+\sum_{j=1}^{r} \lambda_{j}(x) G^{j}[\mathbf{y}]_{\gamma}^{\alpha, \beta}(x)$.

Proof. Suppose that $\mathbf{y}=\left(y_{1}, \ldots, y_{N}\right)$ is the solution to problem defined by (4.1), (4.2), and (4.13). Let $\mathbf{h}=\left(h_{1}, \ldots, h_{N}\right)$ be an arbitrary admissible variation, i.e., $h_{i}(a)=h_{i}(b)=0, i=1, \ldots, N$, and $G^{j}[\mathbf{y}+\varepsilon \mathbf{h}]_{\gamma}^{\alpha, \beta}(x)=0, j=1, \ldots, r$, where $\varepsilon \in \mathbb{R}$ is a small parameter. Because $\mathbf{y}=\left(y_{1}, \ldots, y_{N}\right)$ is a solution to problem defined by (4.1), (4.2), and (4.13), it follows that

$$
\left.\frac{\partial}{\partial \varepsilon} \mathcal{J}(\mathbf{y}+\varepsilon \mathbf{h})\right|_{\varepsilon=0}=0,
$$

that is,

$$
\int_{a}^{b}\left[\sum_{i=2}^{N+1} \partial_{i} L[\mathbf{y}]_{\gamma}^{\alpha, \beta}(x) h_{i-1}(x)+\sum_{i=2}^{N+1} \partial_{N+i} L[\mathbf{y}]_{\gamma}^{\alpha, \beta}(x)^{C} D_{\gamma_{i-1}}^{\alpha_{i-1}, \beta_{i-1}} h_{i-1}(x)\right] d x=0
$$

and, for $j=1, \ldots, r$,

$$
\sum_{i=2}^{N+1} \partial_{i} G^{j}[\mathbf{y}]_{\gamma}^{\alpha, \beta}(x) h_{i-1}(x)+\sum_{i=2}^{N+1} \partial_{N+i} G^{j}[\mathbf{y}]_{\gamma}^{\alpha, \beta}(x)^{C} D_{\gamma_{i-1}}^{\alpha_{i-1}, \beta_{i-1}} h_{i-1}(x)=0 .
$$

Multiplying the $j$ th equation of the system (4.15) by the unspecified function $\lambda_{j}(x)$, for all $j=1, \ldots, r$, integrating with respect to $x$, and adding the left-hand sides (all equal to zero for any choice of the $\lambda_{j}$ ) to the integrand of (4.14), we obtain

$$
\begin{aligned}
& \int_{a}^{b}\left[\sum_{i=2}^{N+1}\left(\partial_{i} L[\mathbf{y}]_{\gamma}^{\alpha, \beta}(x)+\sum_{j=1}^{r} \lambda_{j}(x) \partial_{i} G^{j}[\mathbf{y}]_{\gamma}^{\alpha, \beta}(x)\right) h_{i-1}(x)\right. \\
& \left.\quad+\sum_{i=2}^{N+1}\left(\partial_{N+i} L[\mathbf{y}]_{\gamma}^{\alpha, \beta}(x)+\sum_{j=1}^{r} \lambda_{j}(x) \partial_{N+i} G^{j}[\mathbf{y}]_{\gamma}^{\alpha, \beta}(x)\right)\left({ }^{C} D_{\gamma_{i-1}}^{\alpha_{i-1}, \beta_{i-1}} h_{i-1}(x)\right)\right] d x \\
& =\int_{a}^{b}\left[\sum_{i=2}^{N+1} \partial_{i} F[\mathbf{y}, \lambda]_{\gamma}^{\alpha, \beta}(x) h_{i-1}(x)+\sum_{i=2}^{N+1} \partial_{N+i} F[\mathbf{y}, \lambda]_{\gamma}^{\alpha, \beta}(x)\left({ }^{C} D_{\gamma_{i-1}}^{\alpha_{i-1}, \beta_{i-1}} h_{i-1}(x)\right)\right] d x \\
& =0
\end{aligned}
$$


where $F[\mathbf{y}, \lambda]_{\gamma}^{\alpha, \beta}(x)=L[\mathbf{y}]_{\gamma}^{\alpha, \beta}(x)+\sum_{j=1}^{r} \lambda(x) G^{j}[\mathbf{y}]_{\gamma}^{\alpha, \beta}(x)$. Integrating by parts,

$$
\int_{a}^{b}\left[\sum_{i=2}^{N+1} \partial_{i} F[\mathbf{y}, \lambda]_{\gamma}^{\alpha, \beta}(x)+D_{1-\gamma_{i-1}}^{\beta_{i-1}, \alpha_{i-1}} \partial_{N+i} F[\mathbf{y}, \lambda]_{\gamma}^{\alpha, \beta}(x)\right] h_{i-1}(x) d x=0 .
$$

Because of (4.15), we cannot regard the $N$ functions $h_{1}, \ldots, h_{N}$ as being free for arbitrary choice. There is a subset of $r$ of these functions whose assignment is restricted by the assignment of the remaining $(N-r)$. We can assume, without loss of generality, that $h_{1}, \ldots, h_{r}$ are the functions of the set whose dependence upon the choice of the arbitrary $h_{r+1}, \ldots, h_{N}$ is governed by (4.15). We now assign the functions $\lambda_{1}, \ldots, \lambda_{r}$ to be the set of $r$ functions that make vanish (for all $x$ between $a$ and $b$ ) the coefficients of $h_{1}, \ldots, h_{r}$ in the integrand of (4.16). That is, $\lambda_{1}, \ldots, \lambda_{r}$ are chosen so as to satisfy

$$
\partial_{i} F[\mathbf{y}, \lambda]_{\gamma}^{\alpha, \beta}(x)+D_{1-\gamma_{i-1}}^{\beta_{i-1}, \alpha_{i-1}} \partial_{N+i} F[\mathbf{y}, \lambda]_{\gamma}^{\alpha, \beta}(x)=0, \quad i=2, \ldots, r+1, \quad x \in[a, b] .
$$

With this choice (4.16) gives

$$
\int_{a}^{b}\left[\sum_{i=r+2}^{N+1} \partial_{i} F[\mathbf{y}, \lambda]_{\gamma}^{\alpha, \beta}(x)+D_{1-\gamma_{i-1}}^{\beta_{i-1}, \alpha_{i-1}} \partial_{N+i} F[\mathbf{y}, \lambda]_{\gamma}^{\alpha, \beta}(x)\right] h_{i-1}(x) d x=0 .
$$

Since the functions $h_{r+1}, \ldots, h_{N}$ are arbitrary, we may employ the fundamental lemma of the calculus of variations to conclude that

$$
\partial_{i} F[\mathbf{y}, \lambda]_{\gamma}^{\alpha, \beta}(x)+D_{1-\gamma_{i-1}}^{\beta_{i-1}, \alpha_{i-1}} \partial_{N+i} F[\mathbf{y}, \lambda]_{\gamma}^{\alpha, \beta}(x)=0,
$$

$i=r+1, \ldots, N+1$, for all $x \in[a, b]$. प

REMARK 4.10. In order to determine the $(N+r)$ unknown functions $y_{1}, \ldots, y_{n}$, $\lambda_{1}, \ldots, \lambda_{r}$, we must consider the system of $(N+r)$ equations, consisting of (4.13), (4.17), and (4.18), together with the $2 N$ boundary conditions (4.2).

Assume now that the constraints, instead of (4.13), are characterized by inequalities:

$$
G^{j}[\mathbf{y}]_{\gamma}^{\alpha, \beta}(x) \leq 0, \quad j=1, \ldots, r
$$

In this case we can set

$$
G^{j}[\mathbf{y}]_{\gamma}^{\alpha, \beta}(x)+\left(\phi_{j}(x)\right)^{2}=0, \quad j=1, \ldots, r,
$$

where $\phi_{j}$ have the some continuity properties as $y_{i}$. Therefore, we obtain the following problem:

$$
\hat{\mathcal{J}}(y)=\int_{a}^{b} \hat{L}\left(x, \mathbf{y}(x),{ }^{C} D_{\gamma}^{\alpha, \beta} \mathbf{y}(x), \phi_{1}(x), \ldots, \phi_{r}(x)\right) d x \longrightarrow \min
$$

subject to $r$ independent constraints $(r<N)$

$$
G^{j}[\mathbf{y}]_{\gamma}^{\alpha, \beta}(x)+\left(\phi_{j}(x)\right)^{2}=0, \quad j=1, \ldots, r,
$$

and boundary conditions (4.2). Applying Theorem 4.9 we get the following result.

THEOREM 4.11. A set of functions $y_{1}, \ldots, y_{N}, \phi_{1}, \ldots, \phi_{r}$, which is a solution to problem (4.19) -(4.20), satisfies, for suitably chosen $\lambda_{j}, j=1, \ldots, r$, the following 
system of equations:

$$
\begin{aligned}
D_{1-\gamma_{i-1}}^{\beta_{i-1}, \alpha_{i-1}} \partial_{N+i} \hat{F}\left(x, \mathbf{y}(x),{ }^{C} D_{\gamma}^{\alpha, \beta} \mathbf{y}(x), \lambda_{1}(x), \ldots, \lambda_{r}(x), \phi_{1}(x), \ldots, \phi_{r}(x)\right) \\
+\partial_{i} \hat{F}\left(x, \mathbf{y}(x),{ }^{C} D_{\gamma}^{\alpha, \beta} \mathbf{y}(x), \lambda_{1}(x), \ldots, \lambda_{r}(x), \phi_{1}(x), \ldots, \phi_{r}(x)\right)=0,
\end{aligned}
$$

$i=2, \ldots, N+1$, where $\hat{F}=\hat{L}+\sum_{j=1}^{r} \lambda_{j}\left(G^{j}+\phi_{j}^{2}\right)$, and $\lambda_{j}(x) \phi_{j}(x)=0, j=1, \ldots, r$, hold for all $x \in[a, b]$.

4.5. Sufficient condition of optimality. In this section we provide sufficient optimality conditions for the elementary and the isoperimetric problem of the ${ }^{C} D_{\gamma}^{\alpha, \beta}$ fractional calculus of variations. Similarly to what happens in the classical calculus of variations, some conditions of convexity are in order.

Definition 4.12. Given a function $f \in C^{1}\left([a, b] \times \mathbb{R}^{2 N} ; \mathbb{R}\right)$, we say that $f(\underline{x}, \mathbf{y}, \mathbf{v})$ is jointly convex in $(\mathbf{y}, \mathbf{v})$, if

$$
f\left(x, \mathbf{y}+\mathbf{y}^{0}, \mathbf{v}+\mathbf{v}^{0}\right)-f(x, \mathbf{y}, \mathbf{v}) \geq \sum_{i=2}^{N+1} \partial_{i} f(x, \mathbf{y}, \mathbf{v}) y_{i-1}^{0}+\sum_{i=2}^{N+1} \partial_{N+i} f(x, \mathbf{y}, \mathbf{v}) v_{i-1}^{0}
$$

for all $(x, \mathbf{y}, \mathbf{v}),\left(x, \mathbf{y}+\mathbf{y}^{0}, \mathbf{v}+\mathbf{v}^{0}\right) \in[a, b] \times \mathbb{R}^{2 N}$.

THEOREM 4.13. Let $L(\underline{x}, \mathbf{y}, \mathbf{v})$ be jointly convex in $(\mathbf{y}, \mathbf{v})$. If $\mathbf{y}$ satisfies the system of $N$ fractional Euler-Lagrange equations (4.3), then $\mathbf{y}$ is a global minimizer to problem (4.1)-(4.2).

Proof. The proof is similar to the proof of Theorem 3.3 in 28 . $\square$

Theorem 4.14. Let $F(\underline{x}, \mathbf{y}, \mathbf{v}, \bar{\lambda})=L(\underline{x}, \mathbf{y}, \mathbf{v})-\sum_{j=1}^{r} \bar{\lambda}_{j} G^{j}(\underline{x}, \mathbf{y}, \mathbf{v})$ be jointly convex in $(\mathbf{y}, \mathbf{v})$, for some constants $\bar{\lambda}_{j} \in \mathbb{R}, j=1, \ldots, r$. If $\mathbf{y}^{0}$ satisfies the system of $N$ fractional Euler-Lagrange equations (4.10), then $\mathbf{y}^{0}$ is a minimizer to the isoperimetric problem defined by (4.1), (4.2) and (4.8).

Proof. By Theorem 4.13, $\mathbf{y}^{0}$ minimizes $\int_{a}^{b} F_{\bar{\lambda}}\{\mathbf{y}\}_{\gamma}^{\alpha, \beta}(x) d x$. That is, for all functions satisfying condition (4.2) we have

$$
\begin{aligned}
\int_{a}^{b} L[\mathbf{y}]_{\gamma}^{\alpha, \beta}(x) d x-\sum_{j=1}^{r} \bar{\lambda}_{j} \int_{a}^{b} G^{j}[\mathbf{y}]_{\gamma}^{\alpha, \beta}(x) d x & \\
& \geq \int_{a}^{b} L\left[\mathbf{y}^{0}\right]_{\gamma}^{\alpha, \beta}(x) d x-\sum_{j=1}^{r} \bar{\lambda}_{j} \int_{a}^{b} G^{j}\left[\mathbf{y}^{0}\right]_{\gamma}^{\alpha, \beta}(x) d x .
\end{aligned}
$$

Restricting to the isoperimetric constraints (4.8), we obtain that

$$
\int_{a}^{b} L[\mathbf{y}]_{\gamma}^{\alpha, \beta}(x) d x-\sum_{j=1}^{r} \bar{\lambda}_{j} l_{j} d x \geq \int_{a}^{b} L\left[\mathbf{y}^{0}\right]_{\gamma}^{\alpha, \beta}(x) d x-\sum_{j=1}^{r} \bar{\lambda}_{j} l_{j} d x .
$$

Therefore,

$$
\int_{a}^{b} L[\mathbf{y}]_{\gamma}^{\alpha, \beta}(x) d x \geq \int_{a}^{b} L\left[\mathbf{y}^{0}\right]_{\gamma}^{\alpha, \beta}(x) d x
$$

as desired.

Choosing $r=1$ in Theorem 4.14 one can easily obtain [4, Theorem 3.10]. 
5. Multiobjective fractional optimization. Multiobjective optimization is a natural extension of the traditional optimization of a single-objective function. If the objective functions are commensurate, minimizing one-objective function minimizes all criteria and the problem can be solved using tradicional optimization techniques. However, if the objective functions are incommensurate, or competing, then the minimization of one objective function requires a compromise in another objective. Here we consider multiobjective fractional variational problems with a finite number $d \geq 1$ of objective (cost) functionals

$$
\left(\mathcal{J}^{1}(\mathbf{y}), \ldots, \mathcal{J}^{d}(\mathbf{y})\right)=\left(\int_{a}^{b} L^{1}[\mathbf{y}]_{\gamma}^{\alpha, \beta}(x) d x, \ldots, \int_{a}^{b} L^{d}[\mathbf{y}]_{\gamma}^{\alpha, \beta}(x) d x\right) \longrightarrow \min
$$

subject to the boundary conditions

$$
\mathbf{y}(a)=\mathbf{y}^{a}, \quad \mathbf{y}(b)=\mathbf{y}^{b},
$$

$\mathbf{y}^{a}, \mathbf{y}^{b} \in \mathbb{R}^{N}$, and $r(r<N)$ independent constraints

$$
G^{j}[\mathbf{y}]_{\gamma}^{\alpha, \beta}(x) \leq 0, \quad j=1, \ldots, r,
$$

where $L^{i}, G^{j} \in C^{1}\left([a, b] \times \mathbb{R}^{2 N} ; \mathbb{R}\right), i=1, \ldots, d, j=1, \ldots, r$. We would like to find a function $\mathbf{y} \in \mathbf{D}$, satisfying constraints (5.2) and (5.3), that renders the minimum value to each functional $\mathcal{J}^{i}, i=1, \ldots, d$, simultaneously. The competition between objectives gives rise to the necessity of distinguish between the difference of multiobjective optimization and traditional single-objective optimization. Competition causes the lack of complete order for multiobjective optimization problems. The concept of Pareto optimality is therefore used to characterize a solution to the multiobjective optimization problem. For the usefulness of variational analysis and Pareto optimal allocations in welfare economics, we refer the reader to [32. We define

$$
\mathcal{E}:=\{\mathbf{y} \in \mathbf{D}: \mathbf{y} \text { satisyies conditions (5.2) and (5.3) }\}
$$

Definition 5.1. A function $\overline{\mathbf{y}} \in \mathcal{E}$ is called a Pareto optimal solution to problem (5.1) -(5.3) if does not exist $\mathbf{y} \in \mathcal{E}$ with

$$
\forall i \in\{1, \ldots, d\}: \mathcal{J}^{i}(\mathbf{y}) \leq \mathcal{J}^{i}(\overline{\mathbf{y}}) \quad \wedge \quad \exists i \in\{1, \ldots, d\}: \mathcal{J}^{i}(\mathbf{y})<\mathcal{J}^{i}(\overline{\mathbf{y}}) .
$$

Definition 5.1 introduces the notion of global Pareto optimality. Another important concept is the one of local Pareto optimality.

Definition 5.2. A function $\overline{\mathbf{y}} \in \mathcal{E}$ is called a local Pareto optimal solution to problem (5.1) (5.3) if there exists $\delta>0$ for which does not exist $\mathbf{y} \in \mathcal{E}$ with $\|\mathbf{y}-\overline{\mathbf{y}}\|_{1, \infty}<\delta$ and

$$
\forall i \in\{1, \ldots, d\}: \mathcal{J}^{i}(\mathbf{y}) \leq \mathcal{J}^{i}(\overline{\mathbf{y}}) \quad \wedge \quad \exists i \in\{1, \ldots, d\}: \mathcal{J}^{i}(\mathbf{y})<\mathcal{J}^{i}(\overline{\mathbf{y}}) .
$$

Naturally, any global Pareto optimal solution is locally Pareto optimal. For enhanced notions of Pareto optimality of constrained multiobjective problems, the reader is referred to [6]. 
5.1. Fractional Pareto optimality conditions. We obtain a sufficient condition for Pareto optimality by modifying the original multiobjective fractional problem (5.1) - (5.3) into the following weighting problem:

$$
\sum_{i=1}^{d} w_{i} \int_{a}^{b} L^{i}[\mathbf{y}]_{\gamma}^{\alpha, \beta}(x) d x \longrightarrow \min
$$

subject to $\mathbf{y} \in \mathcal{E}$, where $w_{i} \geq 0$ for all $i=1, \ldots, d$, and $\sum_{i=1}^{d} w_{i}=1$.

TheOrem 5.3. The solution of the weighting problem (5.4) is Pareto optimal if the weighting coefficients are positive, that is, $w_{i}>0$ for all $i=1, \ldots, d$. Moreover, the unique solution of the weighting problem (5.4) is Pareto optimal.

Proof. Let $\overline{\mathbf{y}} \in \mathcal{E}$ be a solution to problem (5.4) with $w_{i}>0$ for all $i=1, \ldots, d$. Suppose that $\overline{\mathbf{y}}$ is not Pareto optimal. Then, there exists y such that $\mathcal{J}^{i}(\mathbf{y}) \leq \mathcal{J}^{i}(\overline{\mathbf{y}})$ for all $i=1, \ldots, d$ and $\mathcal{J}^{j}(\mathbf{y})<\mathcal{J}^{j}(\overline{\mathbf{y}})$ for at least one $j$. Since $w_{i}>0$ for all $i=1, \ldots, d$, we have $\sum_{i=1}^{d} w_{i} \mathcal{J}^{i}(\mathbf{y})<\sum_{i=1}^{d} w_{i} \mathcal{J}^{i}(\overline{\mathbf{y}})$. This contradicts the minimality of $\overline{\mathbf{y}}$. Now, let $\overline{\mathbf{y}}$ be the unique solution to (5.4). If $\overline{\mathbf{y}}$ is not Pareto optimal, then $\sum_{i=1}^{d} w_{i} \mathcal{J}^{i}(\mathbf{y}) \leq \sum_{i=1}^{d} w_{i} \mathcal{J}^{i}(\overline{\mathbf{y}})$. This contradicts the uniqueness of $\overline{\mathbf{y}}$.

Therefore, by varying the weights over the unit simplex $\left\{w=\left(w_{1}, \ldots, w_{d}\right): w_{i} \geq\right.$ $\left.0, \sum_{i=1}^{d} w_{i}=1\right\}$ ones obtains, in principle, different Pareto optimal solutions. The next theorem provides a necessary and sufficient condition for Pareto optimality. The result is analogous to that valid for the finite dimensional case (see, e.g., Chapter 3.1 and Chapter 3.3 of [31]).

Theorem 5.4. A function $\overline{\mathbf{y}} \in \mathcal{E}$ is Pareto optimal to problem (5.1)-(5.3) if and only if it is a solution to the scalar fractional variational problem

$$
\int_{a}^{b} L^{i}[\mathbf{y}]_{\gamma}^{\alpha, \beta}(x) d x \longrightarrow \min
$$

subject to $\mathbf{y} \in \mathcal{E}$ and

$$
\int_{a}^{b} L^{j}[\mathbf{y}]_{\gamma}^{\alpha, \beta}(x) d x \leq \int_{a}^{b} L^{j}[\overline{\mathbf{y}}]_{\gamma}^{\alpha, \beta}(x) d x, \quad j=1, \ldots, d, \quad j \neq i,
$$

for each $i=1, \ldots, d$.

Proof. Suppose that $\overline{\mathbf{y}}$ is Pareto optimal. Then $\overline{\mathbf{y}} \in \mathcal{C}_{k}=\left\{\mathbf{y} \in \mathcal{E}: \mathcal{J}^{j}(\mathbf{y}) \leq\right.$ $\left.\mathcal{J}^{j}(\overline{\mathbf{y}}), j=1, \ldots, d, j \neq k\right\}$ for all $k$, so $\mathcal{C}_{k} \neq \emptyset$. If $\overline{\mathbf{y}}$ does not minimize $\mathcal{J}^{k}(\mathbf{y})$ on the constrained set $\mathcal{C}_{k}$ for some $k$, then there exists $\mathbf{y} \in \mathcal{E}$ such that $\mathcal{J}^{k}(\mathbf{y})<\mathcal{J}^{k}(\overline{\mathbf{y}})$ and $\mathcal{J}^{j}(\mathbf{y}) \leq \mathcal{J}^{j}(\overline{\mathbf{y}})$ for all $j \neq k$. This contradicts the Pareto optimality of $\overline{\mathbf{y}}$. Now, suppose that $\overline{\mathbf{y}}$ minimize each $\mathcal{J}^{k}(\mathbf{y})$ on the constrained set $\mathcal{C}_{k}$. If $\overline{\mathbf{y}}$ is not Pareto optimal, then there exists $\mathbf{y}$ such that $\mathcal{J}^{i}(\mathbf{y}) \leq \mathcal{J}^{i}(\overline{\mathbf{y}})$ for all $i=1, \ldots, d$ and $\mathcal{J}^{j}(\mathbf{y})<\mathcal{J}^{j}(\overline{\mathbf{y}})$ for at least one $j$. This contradicts the minimality of $\mathbf{y}$ for $\mathcal{J}^{j}(\mathbf{y})$ on $\mathcal{C}_{j}$. प

Remark 5.5. For a function $\mathbf{y} \in \mathcal{E}$ to be Pareto optimal to problem (5.1)-(5.3), it is necessary to be a solution to the fractional isoperimetric problems

$$
\int_{a}^{b} L^{i}[\mathbf{y}]_{\gamma}^{\alpha, \beta}(x) d x \longrightarrow \min
$$

subject to $\mathbf{y} \in \mathcal{E}$ and

$$
\int_{a}^{b} L^{j}[\mathbf{y}]_{\gamma}^{\alpha, \beta}(x) d x=\int_{a}^{b} L^{j}[\overline{\mathbf{y}}]_{\gamma}^{\alpha, \beta}(x) d x, \quad j=1, \ldots, d, \quad j \neq i,
$$


for all $i=1, \ldots, d$. Therefore, necessary optimality conditions for the fractional isoperimetric problems (see Theorem 4.8) are also necessary for fractional Pareto optimality.

5.2. Examples. We illustrate our results with two multiobjective fractional variational problems.

ExAMPLE 1. Let $\bar{y}(x)=E_{\alpha}\left(x^{\alpha}\right), x \in[0,1]$, where $E_{\alpha}$ is the Mittag-Leffler function:

$$
E_{\alpha}(z)=\sum_{k=1}^{\infty} \frac{z^{k}}{\Gamma(\alpha k+1)}, \quad z \in \mathbb{R}, \quad \alpha>0 .
$$

When $\alpha=1$, the Mittag-Leffler function is simply the exponential function: $E_{1}(x)=$ $\mathrm{e}^{x}$. We note that the left Caputo fractional derivative of $\bar{y}$ is $\bar{y}$ (cf. [21], p. 98):

$$
{ }_{0}^{C} D_{x}^{\alpha} \bar{y}(x)=\bar{y}(x) .
$$

Consider the following multiobjective fractional variational problem $(N=1, \gamma=1$, and $d=2$ ):

$$
\left(\mathcal{J}^{1}(y), \mathcal{J}^{2}(y)\right)=\left(\int_{0}^{1}\left({ }_{0}^{C} D_{x}^{\alpha} y(x)\right)^{2} d x, \int_{0}^{1} \bar{y}(x)_{0}^{C} D_{x}^{\alpha} y(x) d x\right) \longrightarrow \min
$$

subject to

$$
y(0)=0, \quad y(1)=E_{\alpha}(1)
$$

Observe that $\bar{y}$ satisfies the necessary Pareto optimality conditions (see Remark [5.5). Indeed, as shown in [4. Example 1], $\bar{y}$ is a solution to the isoperimetric problem

$$
\mathcal{J}^{1}(y)=\int_{0}^{1}\left({ }_{0}^{C} D_{x}^{\alpha} y(x)\right)^{2} d x \longrightarrow \min
$$

subject to

$$
\int_{0}^{1} \bar{y}(x)_{0}^{C} D_{x}^{\alpha} y(x) d x=\int_{0}^{1}(\bar{y}(x))^{2} d x .
$$

Consider now the following fractional isoperimetric problem:

$$
\mathcal{J}^{2}(y)=\int_{0}^{1} \bar{y}(x)_{0}^{C} D_{x}^{\alpha} y(x) d x \longrightarrow \min
$$

subject to

$$
\int_{0}^{1}\left({ }_{0}^{C} D_{x}^{\alpha} y(x)\right)^{2} d x=\int_{0}^{1}\left({ }_{0}^{C} D_{x}^{\alpha} \bar{y}(x)\right)^{2} d x .
$$

Let us apply Theorem 4.7. The equality ${ }_{x} D_{1}^{\alpha} y(x)=0$ holds if and only if $y(x)=$ $d(1-x)^{\alpha-1}$ with $d \in \mathbb{R}$ (see [21, Corollary 2.1]). Hence, $\bar{y}$ does not satisfy equation ${ }_{x} D_{1}^{\alpha}\left({ }_{0}^{C} D_{x}^{\alpha} y\right)=0$. The augmented function is

$$
F_{\lambda}\{\mathbf{y}\}_{\gamma}^{\alpha, \beta}(x)=\bar{y}(x)_{0}^{C} D_{x}^{\alpha} y(x)-\lambda\left({ }_{0}^{C} D_{x}^{\alpha} y(x)\right)^{2},
$$


and the corresponding fractional Euler-Lagrange equation gives

$$
{ }_{x} D_{1}^{\alpha}\left(\bar{y}(x)-2 \lambda_{0}^{C} D_{x}^{\alpha} y(x)\right)=0 .
$$

A solution to this equation is $\lambda=\frac{1}{2}$ and $y=\bar{y}$. Therefore, by Remark 5.5. $y=\bar{y}$ is a candidate Pareto optimal solution to problem (5.5) -(5.6).

EXAMPLE 2. Consider the following multiobjective fractional variational problem:

$$
\left(\mathcal{J}^{1}(y), \mathcal{J}^{2}(y)\right)=\left(\int_{0}^{1} \frac{1}{2}\left({ }_{0}^{C} D_{x}^{\alpha} y(x)-f(x)\right)^{2} d x, \int_{0}^{1} \frac{1}{2}\left({ }_{0}^{C} D_{x}^{\alpha} y(x)\right)^{2} d x\right) \longrightarrow \min
$$

subject to

$$
y(0)=0, \quad y(1)=\chi, \quad \chi \in \mathbb{R},
$$

where $f$ is a fixed function. In this case we have $N=1, \gamma=1$, and $d=2$. By Theorem 5.3. Pareto optimal solutions to problem (5.7)-(5.8) can be found by considering the family of problems

$$
w \int_{0}^{1} \frac{1}{2}\left({ }_{0}^{C} D_{x}^{\alpha} y(x)-f(x)\right)^{2} d x+(1-w) \int_{0}^{1} \frac{1}{2}{ }^{C}{ }^{0} D_{x}^{\alpha} y(x) d x \longrightarrow \min
$$

subject to

$$
y(0)=0, \quad y(1)=\chi, \quad \chi \in \mathbb{R},
$$

where $w \in[0,1]$. Let us now fix $w$. By Theorem 4.6, a solution to problem (5.9) -(5.10) satisfies the fractional Euler-Lagrange equation

$$
{ }_{x} D_{1}^{\alpha}\left({ }_{0}^{C} D_{x}^{\alpha} y(x)-w f(x)\right)=0 .
$$

Moreover, by Theorem 4.13, a solution to (5.11) is a global minimizer to problem (5.9) -(5.10). Therefore, solving equation (5.11) for $w \in[0,1]$, we are able to obtain Pareto optimal solutions to problem (5.7) -(5.8). In order to solve equation (5.11), firstly we use Corollary 2.1 of [21] to get the following equation:

$$
{ }_{0}^{C} D_{x}^{\alpha} y(x)-w f(x)=d(1-x)^{\alpha-1}, \quad d \in \mathbb{R} .
$$

Equation (5.12) needs to be solved numerically. We did numerical simulations using the MatLab solver fode for linear Fractional-Order Differential Equations (FODE) with constant coefficients, developed by Farshad Merrikh Bayat [29]. The results for $\alpha=1 / 2, f(x)=\mathrm{e}^{x}$, and different values of the parameter $w$ can be seen in Figure 5.1. Numerical results for different values of $\alpha$ show that when $\alpha \rightarrow 1$ the fractional solution converges to the solution of the classical problem of the calculus of variations.

Acknowledgments. The authors would like to express their gratitude to Ivo Petras, for having called their attention to [29] as well as for helpful discussions on numerical aspects and available software packages for fractional differential equations.

\section{REFERENCES}

[1] R. Almeida, A. B. Malinowska and D. F. M. Torres, A fractional calculus of variations for multiple integrals with application to vibrating string, J. Math. Phys. 51 (2010), no. 3, 033503, 12 pp. arXiv: 1001.2722 


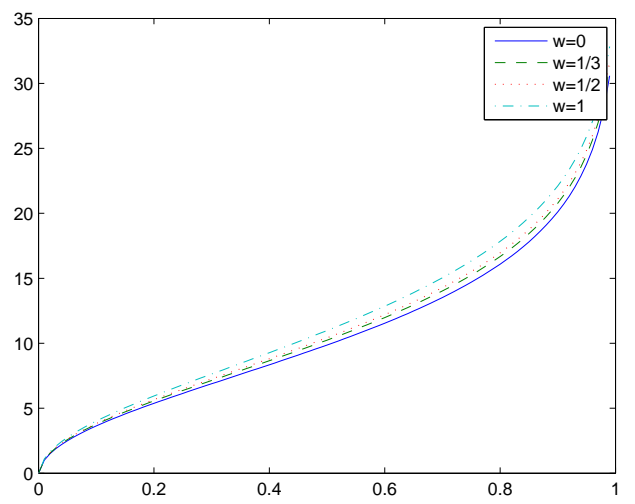

FIG. 5.1. The minimizer of Example Q for $f(x)=\mathrm{e}^{x}$ and $\alpha=\frac{1}{2}$.

[2] R. Almeida And D. F. M. Torres, Calculus of variations with fractional derivatives and fractional integrals, Appl. Math. Lett. 22 (2009), no. 12, 1816-1820. arXiv:0907.1024

[3] R. Almeida and D. F. M. Torres, Leitmann's direct method for fractional optimization problems, Appl. Math. Comput. 217 (2010), no. 3, 956-962. arXiv:1003.3088

[4] R. Almeida and D. F. M. Torres, Necessary and sufficient conditions for the fractional calculus of variations with Caputo derivatives, Commun. Nonlinear Sci. Numer. Simul. 16 (2011), no. 3, 1490-1500. arXiv:1007.2937

[5] T. M. Atanacković, S. Konjik and S. Pilipović, Variational problems with fractional derivatives: Euler-Lagrange equations, J. Phys. A 41 (2008), no. 9, 095201, 12 pp. arXiv:1101.2961

[6] T. Q. Bao And B. S. Mordukhovich, Relative Pareto minimizers for multiobjective problems: existence and optimality conditions, Math. Program. 122 (2010), no. 2, Ser. A, 301-347.

[7] N. R. O. Bastos, R. A. C. Ferreira and D. F. M. Torres, Necessary optimality conditions for fractional difference problems of the calculus of variations, Discrete Contin. Dyn. Syst. 29 (2011), no. 2, 417-437. arXiv:1007.0594

[8] N. R. O. Bastos, R. A. C. Ferreira And D. F. M. Torres, Discrete-time fractional variational problems, Signal Process. 91 (2011), no. 3, 513-524. arXiv:1005.0252

[9] L. Debnath, Recent applications of fractional calculus to science and engineering, Int. J. Math. Math. Sci. 2003, no. 54, 3413-3442.

[10] K. Diethelm and A. D. Freed, On the solution of nonlinear fractional-order differential equations used in the modeling of viscoplasticity. In: F. Keil, W. Mackens, H. Voß, J. Werther (eds.): Scientific Computing in Chemical Engineering II. Computational Fluid Dynamics, Reaction Engineering, and Molecular Properties. Springer-Verlag, Heidelberg, 1999, 217-224.

[11] R. A. El-Nabulsi And D. F. M. Torres, Necessary optimality conditions for fractional actionlike integrals of variational calculus with Riemann-Liouville derivatives of order $(\alpha, \beta)$, Math. Methods Appl. Sci. 30 (2007), no. 15, 1931-1939. arXiv:math-ph/0702099

[12] R. A. El-Nabulsi and D. F. M. TorRes, Fractional actionlike variational problems, J. Math. Phys. 49 (2008), no. 5, 053521, 7 pp. arXiv:0804.4500

[13] J. Engwerda, Necessary and sufficient conditions for Pareto optimal solutions of cooperative differential games, SIAM J. Control Optim. 48 (2010), no. 6, 3859-3881.

[14] N. M. Fonseca Ferreira, F. B. Duarte, M. F. M.Lima, M. G. Marcos and J. A. TenREIRo MACHADO, Application of fractional calculus in the dynamical analysis and control of mechanical manipulators, Fract. Calc. Appl. Anal. 11 (2008), no. 1, 91-113.

[15] G. S. F. Frederico And D. F. M. Torres, A formulation of Noether's theorem for fractional problems of the calculus of variations, J. Math. Anal. Appl. 334 (2007), no. 2, 834-846. arXiv:math/0701187

[16] G. S. F. Frederico And D. F. M. Torres, Fractional conservation laws in optimal control theory, Nonlinear Dynam. 53 (2008), no. 3, 215-222. arXiv:0711.0609

[17] G. S. F. Frederico And D. F. M. Torres, Fractional Noether's theorem in the Riesz-Caputo sense, Appl. Math. Comput. 217 (2010), no. 3, 1023-1033. arXiv:1001.4507 
[18] M. Giaquinta and S. Hildebrandt, Calculus of variations. I, Springer, Berlin, 1996.

[19] R. Hilfer, Fractional diffusion based on Riemann-Liouville fractional derivatives, J. Phys. Chem. B 104 (2000), no. 16, 3914-3917.

[20] R. Hilfer, Applications of fractional calculus in physics, World Sci. Publishing, River Edge, NJ, 2000.

[21] A. A. Kilbas, H. M. SRivastava And J. J. Trujillo, Theory and applications of fractional differential equations, Elsevier, Amsterdam, 2006.

[22] M. KLIMEK, Stationarity-conservation laws for fractional differential equations with variable coefficients, J. Phys. A 35 (2002), no. 31, 6675-6693.

[23] V. V. Kulish And J. L. Lage, Application of fractional calculus to fluid mechanics, J. Fluids Eng. 124 (2002), no. 3, 803-806.

[24] E. R. Love and L. C. Young, On fractional integration by parts, Proc. London Math. Soc. 44 (1938), no. 2, 1-35.

[25] R. Magin, Fractional calculus in bioengineering. Part 1-3, Critical Reviews in Bioengineering, 32 (2004).

[26] A. B. Malinowska and D. F. M. Torres, Nonessential functionals in multiobjective optimal control problems, Proc. Estonian Acad. Sci. Phys. Math. 56 (2007), no. 4, 336-346. arXiv:math/0609731

[27] A. B. Malinowska And D. F. M. Torres, Computational approach to essential and nonessential objective functions in linear multicriteria optimization, J. Optim. Theory Appl. 139 (2008), no. 3, 577-590. arXiv:0706.1192

[28] A. B. Malinowska and D. F. M. Torres, Generalized natural boundary conditions for fractional variational problems in terms of the Caputo derivative, Comput. Math. Appl. 59 (2010), no. 9, 3110-3116. arXiv:1002.3790

[29] F. Merrikh-Bayat, Fractional-order differential order equation solver, Matlab Central, File ID: \#13866, 2007. http://www.mathworks.com/matlabcentral/fileexchange/13866

[30] F. Metzler, W. Schick, H. G. Kilian and T. F. Nonnenmacher, Relaxation in filled polymers: a fractional calculus approach, J. Chem. Phys. 103 (1995), 7180-7186.

[31] K. Miettinen, Nonlinear multiobjective optimization, Kluwer Acad. Publ., Boston, MA, 1999.

[32] B. S. Mordukhovich, Nonlinear prices in nonconvex economies with classical Pareto and strong Pareto optimal allocations, Positivity 9 (2005), no. 3, 541-568.

[33] B. S. Mordukhovich, Variational analysis and generalized differentiation. II, Grundlehren der Mathematischen Wissenschaften, 331, Springer, Berlin, 2006.

[34] D. Mozyrska and D. F. M. Torres, Minimal modified energy control for fractional linear control systems with the Caputo derivative, Carpathian J. Math. 26 (2010), no. 2, 210-221. arXiv: 1004.3113

[35] D. Mozyrska And D. F. M. Torres, Modified optimal energy and initial memory of fractional continuous-time linear systems, Signal Process. 91 (2011), no. 3, 379-385. arXiv:1007.3946

[36] K. B. Oldham and J. Spanier, The fractional calculus, Academic Press [A subsidiary of Harcourt Brace Jovanovich, Publishers], New York, 1974.

[37] A. Oustaloup, V. Pommier and P. Lanusse, Design of a fractional control using performance contours. Application to an electromechanical system, Fract. Calc. Appl. Anal. 6 (2003), no. $1,1-24$.

[38] I. Podlubny, Fractional differential equations, Academic Press, San Diego, CA, 1999.

[39] F. Riewe, Nonconservative Lagrangian and Hamiltonian mechanics, Phys. Rev. E (3) 53 (1996), no. 2, 1890-1899.

[40] F. RIEwe, Mechanics with fractional derivatives, Phys. Rev. E (3) 55 (1997), no. 3, part B, 3581-3592.

[41] B. Ross, S. G. Samko And E. R. Love, Functions that have no first order derivative might have fractional derivatives of all orders less than one, Real Anal. Exchange 20 (1994/95), no. $1,140-157$.

[42] S. G. Samko, A. A. Kilbas and O. I. Marichev, Fractional integrals and derivatives, Translated from the 1987 Russian original, Gordon and Breach, Yverdon, 1993.

[43] A. Sophos, E. Rotstein and G. Stephanopoulos, Multiobjective analysis in modeling the petrochemical industry, Chem. Eng. Sci. 35 (1980), 2415-2426.

[44] J. A. Tenreiro Machado and R. S. Barbosa, Introduction to the special issue on "Fractional Differentiation and its Applications", J. Vib. Control 14 (2008), no. 9-10, 1253.

[45] J. L. Troutman, Variational calculus and optimal control, Springer, New York, 1996.

[46] F.-S. WANG AND T.-L. Shieh, Extension of iterative dynamic programming to multiobjective optimal control problems, Ind. Eng. Chem. Res. 36 (1997), 2279-2286.

[47] G. M. Zaslavsky, Hamiltonian chaos and fractional dynamics, Oxford Univ. Press, Oxford, 2008. 